\title{
Bestimmung der Atomgewichte von Cäsium und Rubidium;
}

von Richard Godeffroy.

(Eingelaufen den 5. Januar 1876.)

Als Material für die nachstehend beschriebenen Versuche diente hauptsächlich ein aus der Fabrik des Herrn Dr. Schuchardt in Görlitz bezogenes sogunanntes reines Chlorcäsium, ferner benutzte ich Lepidolithrückstande und Nauheimer Mutterlaugensalz. Zur Herbeischaffung dieses Materials wurden mir auf meine Bitte von Seiten der chemisch-physikalischen Gesellschaft in Wien $100 \mathrm{fl}$. $\ddot{0}$. W. und von Seiten des allgemeinen österr. Apotheker-Vereins in Wien $200 \mathrm{fl}$. $0 . \mathrm{W}$. gütigst gewährl.

Ich begann danit, die bisher angregebenen Gesinnungsmethoden von Cäsiun und Rubidium einer sorgfältigen Prüfung zu unterwerfen.

Noch heute wird die von $B$ unsen ${ }^{3}$ ) angegebere Methode zur Trennung von Cäsium und Rubidium von einander ind von den übrigen Alkalimetallen allgemein für die beste und sicherste gehalten. Nach derselben wird der aus Lepidolithen erhaltene Salzrückstand, welcher ans Cilornatrium, Chlorkalium, Chlorrubidium neben geringt'n Mengen von Chlorcäsium und Chlorstrontium besteht, in Wasser gelöst und mit Platinchlorid versetzt, wodurch ein Niederschlag der Platinchloriddoppelsalze des Kaliums, Rubidiuns und Cäsiums entsteht. Dieser Niederschlag wird 20 mal hintereinander mit sehr wenig Wasser gekocht, hitrauf getrocknet und durch $W$ asserstoffgas reducirt. Aus dem Rückstande werden die Alkalisalze mit

*) Diese Annalen 128, 351; Jahresboricht für Chemio u. s. ซ. f. 1861,172 . 
kochendem Wasser ausgezogen. Zur Entfernung des Chlorkaliums wird die märsig verdünnte heifse Lösung mit Platinchlorid gefällt und der Niederschlag mit warmem Wasser ausgewaschen. Um auch dic letzten Kaliumspuren zu beseitigen, soll die Fällung nach jedesmaliger Reduction durch Wasserstoffgas noch zwei- bis dreimal auf dieselbe Weise behandelt werden. Schliefslich erhält man ein chlorcãsiumhaltiges Chlorrubidium, welches im Spectralapparate geprün keine Spur der rothen Kaliumlinie mehr zeigt. Zur Entfernung des kleinen Cäsiumgehaltes empfichlt Bunsen, die Chlorverbindungen in Sulfate zu verwandeln, die Schwefelsäure aus der Lösung durch Zusatz eines geringen Ueberschusses von Barythydrat zu entfernen und die erhaltenen Oxydhydrate mit Ammoniumcarbonat in einer Silberschale zur Trockne einzudampfen. Die vorher durch Filtration von etwas kohlensaurem Baryum getrennten Carbonate werden entwässert und dann 20 bis $30 \mathrm{mal}$ mit kochenden absolutem Alkohol behandelt, wodurch das kohlensaur: Cäsium unter Zurücklassung von reinem kohlensaurem Rubidium gelöst wird.

Diese Methode, welche sich in erster Linie auf die verschiedene Lösliclıkeit der Platindoppelsalze des Kaliums, Rubidiums und Cäsiums in leeifsem Wasser und in zweiter Linie auf die verschiedene Löslichkeit der Carbonate des Rubidiums und Cäsiums in heifsem absolutem Alkokol gründet, wurde auch mit geringer Abweichung von Heintz*), J. Piccard**) und R. Böttger***) empfohlen.

Ich habe diese Methode mit $20 \mathrm{Grm}$. eines Gemenges dor

*) Diese Annalen I84, 129; Jahresber. f. Chemie a. s. w. f. 1862, 121 und $f$. 1865,169 .

**) Joumal für praktiache Chemie es, 449; Jahresber. f. Chemio u. 8. w. f. $1862,123$.

**) Diene Annalen 122,368 und 128,240 ; Jabresber. f. Chemio u. 8. w. f. $1863,185$.

Annaten der Chamie 181. Bd. 
Chloride des Lithiums, Natriums, Kaliums, Rubidiums und Cäsiums, in welchem letzteres vorherrschend war, auszuführen versucht. Wenn es mir auch mit vieler Mühe und grofsem Zeitaufwande gelungen ist, auf diese Weise Rubidium und Cäsium von den übrigen Alkalimetallen zu befreien, so erzielte ich doch niemals eine vollständige Trennung des Rubidiums und Cäsiums von einander, da einerseits dio alkoholische Lösung von Cäsiumcarbonat immer noch Spuren von Rubidium und anderseits der in Alkohol unlösliche Rückstand von Rubidiumcarbonat im Spectralapparate immer noch Spuren von Cäsium zeigten.

A. Schrötter*), welcher fand, dafs Kaliumplatinchlorid sich in verdünnter wässeriger Chlorkaliumlösung weniger löst, als in reinem Wasser, in einer concentrirten aber fast unlöslich ist, empfiehlt, eine Kaliumplatinchloridlōsung zur Fällıng von rubidium- und cäsiumhaltigen Flüssigkeiten zu nehmen, nur müsse man die zu fällende Flüssigkeit vor deın Zusatz der kalt gesättigien Kaliumplatinchloridlösung erhitzen und hierauf mit derselben bis zum Krystallisiren des Chlorkaliums eindampfen, damit das ausgeschiedene Kaliumdoppelsalz sich allmälig in Rubidium- und Cäsiumplatinchlorid umsetze.

Diese Methode der Ausscheidung von Rubidium und Cäsium aus einem Gemenge der Lōsungen der übrigen Alkalimetalle ist wohl nur dann anwendbar, wenn jene beiden Elemente in sehr geringen Mongen vorhanden sind, da bei der geringen Löslichkeit des Kaliumplatinchlorids in $W$ asser sonst ein zu grofses Flüssigkeitsquantum erreicht würde, wclches der vollständigen Ausscheidung des Rubidiums und Cäsiums hinderlich sein dürfte.

*) Wiener Academie-Beriohto SO, 268; Jahresberieht f. Chemio u. s. w. f. $1864,186$. 
0. D. Allen *) gründet seine Trennungsmethode auf die verschiedene Lōslichkeit der sauren Tartrate des Cäsiums und Rubidiums. Die nach Bunsen's Methode erhaltenen kohlensauren Salze der beiden Metalle werden mit dem Doppelten der zur Sättigung nöthigen Menge Weinsäure versetzt und die Lòsung concentrirt, bis sie bei $100^{\circ} \mathrm{C}$. fast gesättigt ist. Beim Abkühlen krystallisirt zuerst das Rubidiumsalz heraus und es kann von diesem das löslichere Cäsiumsalz durch fractionirte Krystallisation getrennt werden, da 1 Theil des sauren weinsauren Rubidiums sich in 8,5 Theilen siedendem Wasser oder in 84,5 Theilen Wasser von $25^{\circ} \mathrm{C}$. löst, während das ähnlich krystallisirende saure weinsaure Cäsium schon in 1,02 Theilen siedendem $W$ asser und in 10,3 Theilen Wasser von $25^{\circ} \mathrm{C}$. löslich ist.

Eine ähnliche Trennungsmethode ist auch von Bunsen **) empfohlen, aber dabei die Eigenschaft des normalen weinsauren Cäsiums, an der Luft zu zerfliefsen, benutzt worden, während das saure weinsqure Rubidium und das saure weinsaure Cäsium luftbeständig sind. Nachdem man in einer Probe des von Kalium, Natrium und Lithium völlig freien Gemenges von Chlorcäsium und Chlorrubidium den Rubidiumgehalt durch eine Chlorbestimmung ermittelt hat, verwandelt man die Chlorverbindungen in Carbonate, setzt der Losung derselben etwas mehr Weinsäure hinzu, als erforderlich ist, um neutrales weinsaures Cäsium und saures weinsaures Rubidium zu erhalten, verdampft zur Trockue und bringt die Salzmasse in einem Trichter, worin sich ein Papierfilterchen befindet, in eine mit Wasserdampf gesättigte Almosphäre.

*) Journal für praktische Chemie 8Q, 82; Jahresber. f. Chemie u. 8. w. f. 1862,122 .

**) Pogg. Annalen 119, 1; Jahresber. f. Chemie u. s. w. f. 1868, 187. 
Das Rubidium bleibt vollständig als saures weinsaures Rubidium auf dem Filter zurück, während eine concentrirte Lösung des normalen weinsauren Cäsiums abtropft.

Lecoq de Boisbaudran*) gewiunt das Cäsium und Rubidium aus Lepidolith auf ähnliche Weise, nur empfiehlt er, anstatt das saure weinsaure Salz zu waschen und auf einem Trichter zu trocknen, dasselbe besser in der nöthigen Menge siedendem Wasser aufzulösen und die Lösung unter Umrühren erkalten zu lassen. Durch zweimaliges Wiederholen dieser Operation soll man völlig cäsiumfreies Rubidiumsalz erhalten.

Ich konnte diese Trennungsmethode nur dann mit Erfolg benutzen, wenn Cäsium nicht in überwiegender Menge vorhanden war, da ich sonst nur mit grofsem Verluste an Material die Tartrate des Cäsiums uud Rubidiums getrennt erhielt.

Im Jahre 1865 hat J. Redtenbacher * ) auf die verschiedene Löslichkeit der Alaune des Kaliums, Rubidiums und Cäsiums eine neue Trennungsmethode dieser Metalle gegründet. Die drei Alaune, welche in heifsern Wasser gleich löslich sind, zeigen nämlich bei $17^{\circ} \mathrm{C}$. einen bedeutenden Unterschied in ihrer Löslichkeit, indem bei dieser Temperatur 100 Theile Wasser von

$\begin{array}{ccc}\text { Kaliumalaun } & \text { Rubidiumalaun } & \text { Cäsiumalann } \\ 13,5 \text { Theile } & 2,27 \text { Theile } & 0,619 \text { Theile }\end{array}$

lösen. Vergleicht man damit die Löslichkeitsverhältnisse der Platindoppelsalze, so ist der Kalialaun 13mal, der Rubidiumalaun 15 mal und der Cäsiumalaun $88 \mathrm{mal}$ löslicher •als die

*) Bulletin de la soc. chim. [2] 17, 551; Jahresber. f. Chemio u. s. w. f. $1872,235$.

**) Wiener acad. Anzeiger f. 1865, 39; Jahresber. f. Chemie u. s. w. f. 1865,704 . 
correspondirenden Platindoppelsalze, da 100 Theile Wasser bei $17^{\circ} \mathrm{C}$. von
Kaliumplatinchlorid Bubidiumplatinchlorid Casiumplatinchlorid 1,12 Theile 0,141 Theile 0,079 Theile

lösen; demnach wird das relative Verhälınifs der Löslichkeit der Alaune und Platinsalze in Wasser von $17^{\circ} \mathrm{C}$. durch nachstehende Zahlen ausgedrückt :

$\begin{array}{lccc} & \text { Kaliumsalz } & \text { Rubidiumsalz } & \text { CHoiumsalz } \\ \text { Alaune : } & 22 & 4 & 1 \\ \text { Platinsalze : } & 15 & 2 & 1 .\end{array}$

Auch hier gelingt es nicht, auf einmal sämmtliches Cäsium von Rubidium und Kalium zu trennen, allein man erhält bei etwas sorgfaltiger fractionirter Krystallisation neben vollkommen reinem Materiale noch Alaune, aus denen sich, wie ich später zeigen werde, das Cäsium vom Rubidium leicht trennen lärst.

F. Stolba ) hat zur Darstellung reiner Rubidium- und Cäsiumverbindungen die von Bunsen und Redtenbacher angegebenen Methoden vereinigt. Er schlägt vor, den Platinchloridnicderschlag auf einem Filter zu sammeln, mit wenig Wasser zu waschen und in siedende Kieselfluorwasserstoffsäure von etwa 6 pC. Säuregehalt einzutragen, dadurch wird das Kaliumplatinchlorid vollständig zersetzt, während diefs bei den schworer löslichen Platinchloriddoppelsalzen des Rubidiums und Cäsiums nicht so leicht vor sich geht; es soll auch nicht zweckuluäsig scin, diese vollständig zu zersetzen. Nach $1 / 4$ stündigem Sieden wird die Flüssigkeit abgegossen, der Rüchstand nochmals einer gleichen Behandlung unterworfen, dann getrockntet und mit concentrirter Schwefelsäure zersetzt. Dem schliefslich geglühten Rückstande werden durch Wasser

*) Journal für praktische Chemio De, 49; Jabresber. f. Chemio u. s. w. f. 1866,151 . 
die Sulfate des Kaliums, Rubidiums und Cäsiums entzogen und letztere in Form von Alaunen getrennt.

Später hat F. Stolba *) sein Verfahren insofern umgeändert, als er empfiehlt, das rubidium- und căsiumhaltige Salz sofort in Alaune zu verwandeln und letztere in einem grofsen Ueberschufs von concentrirter Salzsäure zu lösen. Wird der erkalteten Lösung Zinnchloridlösung hinzugefügt, so entsteht ein massiger krystallinischer Niederschlag von Cäsiumıinnchlorid, w.elcher ıit concentrirter Salzsäure ausgesüfst, dann in salzsäurehaltigem Wasser gelōst und mit concentrirter Salzsäure wieder herausgefällt werden kann und schliefslich ein rubidium- und kaliumfreies Salz liefert. Die von Cäsiumzinnchlorid abfiltrirte Flūssigkeit giebt nach dem Yerdampfen der überschüssigen Salzsãure mit Kieselfluorwasserstoffāure und Alkohol einen Niederschlag von Kieselfluorrubidium, welcher sich durch Glühen mit Sulmiak leicht in Chlorrubidium verwandeln läfst. Auch das Cäsiumzinnchlorid kann durch Glühen mit Salmiak leicht in Chlorcásium ungewandelt werden.

Nachdem ich die eben beschriebenen Trennungsmethoden des Cásiums und Rubidiums von einander und von den übrigen Alkalimetallen sammtlich versucht hatte, glauble ich in den grofsen Löslichkeitsunterschieden der drei Alaune des Kaliums, Rubidiums und Cäsiums, ferner in der Leichtigkeit, mit welcher dieselben zu erhalten sind, den raschesten und sichersten Weg zur Trennung dieser Elemente gefunden zu haben. Deshalb wurde mein ganzes Huuptmaterial auf bekannte Weise in Alaune umgewandelt und bei $17^{\circ} \mathrm{C}$. einer fractionirten Krystallisation unterworfen. Schon die ersten Krystalle, welche ich erhielt, bestanden (spectralanalytisch

*) Dingler's polyt. Journel 108, 225 and 198, 336; Jahresber. f. Chemie u. 8. w. f. 1866,161 und f. 1870,307 and 988 . 
geprüft) hauptsáchlich nur aus Cäsiumalann, welcher gesammelt, mit destillirtem Wasser gewaschen und in siedendem Wasser wieder aufgelöst wurde. Nach zweimaligem Umkrystallisiren und jedesinaligem Auswaschen der Krystalle mit Wasser von $17^{\circ} \mathrm{C}$. war dieser Cäsiumalaun vollkommen rein, wenigstens zeigte er im Spectralapparate nur die bekannten Cäsiumlinien.

Die erhaltenen Mutterlaugen und Waschwasser wurden vereinigt und abermals krystallisiren gelassen. Die nun gebildeten Krystalle wurden wieder gesammelt und wie früher beschrieben umkrystallisirt; es gelang aber erst nach sechsmaligem Umkrystallisiren, eine kleine Menge von spectralanalytisch vollkommen reinem Cäsiumalaun zu erhalten, während die grörste Menge desselben immer noch Spuren ron Rubidium zeigte, die sich durch noch so sorgfältiges Unkrystallisiren nicht entfernen liefsen. Indem ich auf diese Weise fortfahrend immer die sich bildenden Krystalle sammelte, umkrystallisirte und mit Wasser wusch, erhielt ich folgende Reihe von Alaunen :

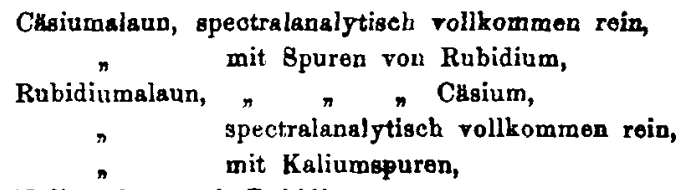

Kaliumalaun, mit Rubidiumspuren.

Um aus dem Cäsiumalaun, welchęr noch Spuren von Rubidium zeigte, das Cäsium vom Rubidium zu trennen, benutzte ich die von mir *) schon mitgetheilte Eigenschaft des Antimontrichlorids, in mit Salzsäure angesäuerten Lősungen von Chlorcasium und Chlorrubidium nur mit ersterem einen in concentrirter Salzsăure unlöslichen Niederschlag von Cäsium-

*) Berichte der deutschen obemischen Gesellechaft $\mathbf{7}, 375$ und $\mathbf{6}, 9$; Zeitschrift des allgem. österr. Apotheker-Vereins f. 1874, Nr. 9 und f. 1875 , Nr. 2. 
antimonchlorid za geben. Demnach bereitete ich mir aus diesem Cäsiumalaun, nach Ausfällung der Thonerde mit Ammoniak, Verjagung des gebildeten Ammoniumsulfats durch Glühen, die Sulfate des Cäsiums und Rubidiums, welche beide auf bekannte Weise in Chloride umgewandelt wurden. Letztere löste ich in concentrirter Salzsäure auf und vermischte die Lōsung mit einer Lösung von Antimontrichlorid in concentrirter Salzsüure. Sofort entstand ein reichlicher Niederschlag von Cäsiumantimonchlorid, weicher auf einem Filter gesammelt und mit concentrirter Salzsäure mehrmals gewaschen wurde; derselbe zeigte, im Spectralapparate geprüft, keine Spur von Rubidium mehr. Wird dieser Niederschlag mit Wasser behandelt, so zersetat er sich unter Ausscheidung von Antimonoxychlorid, wälırend Chlorcäsium mit geringen Mengen von Cäsiumantinonchlorid in Lösung gehen. Um letztere ganz zu entfernen dampft man die Flüssigkeit zur Trockne ein und glüht den Rückstand mit Salmiak, wodurch alles Antimon als Antimonchlorid verflüchtigt werden kann.

Auch der Rubidiumalaun, welcher noch Spuren von Cäsium zeigte, wurde auf die eben beschriebene $W$ eise behandelt, nur mufste der durch Antimontrichlorid entstandene Niederschlag (da er nach dem Aussüfsen mit concentrirter Salzsäure, im Sprctralapparate geprüft, noch die Rubidiumlinien zeigte) in verdünntur Salzsäure gelöst und aus dieser Lōsung das Cäsiumantimonchlorid durch concentrirte Salzsăure und einiges Stehenlassen wieder herausgefällt werden.

Es ist durchaus nicht zweckmärsig, den Alaun direct in Salzsäure zu lösen, wie es F. Slolba bei seiner Trennungsmethode mit Zinnchlorid angiebt, da man sonst bei der viel geringeren Löslichkeit der Alaune in Salzsäure, im Gegensatze zu der der Chloride, ein so grofses Flüssigkeitsquantum erhält, lafs sich die vorhandenen geringen Mengen von Cäsium daraus nicht vollständig fällen lassen. Ich glaube 
auch, das Antimontrichlorid als Trennungsmittel dem Zinnchlorid vorziehen zu dürfen, da letzteres in der concentrirten salzsauren Lösung auch mit Chlorrubidium und Chlorammonium schwer lōsliche Niederschläge erzeugt, wodurch die Trennung des Rubidiums vom Cäsium erschwert wird; ferner ist die Gewinnung des Chlorcäsiums aus Cäsiumantimonchlorid mit weniger Verlust verbunden als aus Cäsiumzinnchlorid, da ersteres schon beim Uebergiefsen mit Wasser beinahe vollständig zersetzt wird und inan zur Vertreibung der letzten Spuren von Antimonchlorid kein so langes Glühen mit Salmiak nöthig hat, was mir bei der grofsen Flüchtigkeit des Chlorcäsiums wichtig genug erscheint.

Atomgewichtsbestimmung des Cäsiums.

Zur Darstellung von vollkommen reinem Chlorcäsium für die Atomgewichtsbestimnung des Cäsiums wurde nur der vollkommen reine Cäsiumalaun verwendet, d. h. jener, welcher im Spectralapparate nur die Cäsiumlinien zeigte.

Dieser Cäsiumalaun wurde in heifsem Wasser *) gelōst, mit Anımoniak *) versetzt, der entstandene Thonerdeniederschlag abfiltrirt, das Filtrat zuerst in einer Platinschale bis zur Trockne verdampft und schliefslich geglüht bis zur Verjagung alles Ammoniumsulfats. Nach dem Erkalten wurde die geschmolzene Masse in Wasser aufgelöst und so lange mit einer Lösung von reinen Chlorbaryum **) in Wasser

*) Beide wurden vorher durch Eindampien ron je $200 \mathrm{CC}$. in einer Platinschale auf einen Gebalt an feuerbeständigen Salzen geprüt.

**) Das zur Fallung benutete Chlorbaryum wurde ans einem kHuflichen sogenannten chemisch reinen Salz durch dreimaliges Um. krystallisiren gewonnen und ror der Verwendung noch auf oinen otwaigen Gebalt an Chlorcalcium, Chlorstrontium und den Alkalimetallchloriden gepruft, indem $20 \mathrm{Grm}$. desselben in 2 Liter Wasser gelöst und so lange mit verdünnter Bchwefelsiure versetzt wurden, als noch ein Niederschlag entutand. Die von letzterem klar ab- 
versetzt, als noch ein Niederschlag von Baryumsulfat entstand; letzterer wurde abfiltrirt und das Filtrat zur Fallung des überschüssig zugesetzten Chlorbaryums und des in der Flüssigkeit etwa noch vorhandenen Thonerdesalzes mit Ammoniak und einer Lösung von reinem Ammoniumcarbonat *) in Wasser versetzt und erwärmt. Nach einigem Stehen wurde die Flüssigkeit vom entstandenen Niederschlag abfiltrirt, hierauf in einer Platinschale zuerst zur Trockne eingedampft and dann geglüht. Die geschmolzene Masse wurde nach dem Erkalten abermals in $W$ asser gelöst, die Lösung von etwa vorbandenem Baryumcarbonat durch Filtration befreit, dann neuerdings mit Ammoniak und Ammoniumcarbonat versetzt, erwärmt und durch einige Tage im bedeckten Gefäfse stehen gelassen, um die letzten Spuren des etwa vorlandenen Thonerdesulfats oder Chlorbaryums zu entfernen. Obschon sich kein Niederschlag zeigte, wurde die Flüssigkeit doch vorsichtshalber filtrirt, hieraul in einer Platinschale zur Trockne eingedampft und schliefslich bis zur Vertreibung aller Ammoniaksalze geglüht und geschmolzen.

Die geschmolzene Masse zeigte keine alkalische Reaction, dieselbe wurde in wenig Wasser gelöst und der Krystallisation überlassen. Die erhaltenen Krystalte waren durchaus nicht zerfliefslich; ich habe eine kleine Menge derselben durch ein volles Jahr im Laboratorium in einem offenen Gefäfse stehen, ohne ein Zerfliefsen beobachtet zu haben.

Die Atomgewichtsbestimmung dieses so dargestellten reinen Chlorcäsiums geschah auf zweierlei Weise, durch Filtration und durch Decantiren in oiner Glasschale.

fltrirte Flüssigkeit gab nach dem rollstandigen Eindampfen in einer Platinschale einen kaum merkbaren Rückstand, welchor im Spectralapparate geprüft nur die bekannten Baryumlinien zeigte.

* Das Ammoniumcarbonat wurdo vorher durch Gltben im Platintiegel and spectralanalytiscbe Untersuchung des kaum merkbaren Rückstandes auf einen Gehalt an Chlorcaloium geprüft. 
1) Durck Filtration. - Eine genau gewogene Menge des Salzes wurde in einem Becherglase in heifsem, durch Salpetersäure ungeskuertem Wasser getosst und das Chlor durch überschtissig zugesetzte Silbernitratlösung gefält. Nachdem sich der entstandene Niederschlag vollständig abgesetzt halte, wurde die Flüssigkeit durch ein vorher mit heifser verdünnter Salpetersäure wiederholt gewaschenes Filter, dessen Aschengehalt genau bestimmt wurde, filtrirt, der Niederschlag anfangs im Bechergluse, dann aber auf dem Filter so lange mit heifsem, durch Salpetersäure angesäuertem Wasser gewaschen, bis eine Probe des Filtrats mit Salzsäure versetzt nicht mehr getrübt wurde. Der Niederschlag wurde nun in Fitter vollstăndig getrocknet, hierauf letzteres vorsichtig vom Niederschlage abgelöst und für sich in einem vorhor genau gewogenen Porcellantiegel eingeäschert, die Asche sodann mit Salpetersalzsäure erhitzt und nach vollständiger Verjagung aller Dämpfe der Chlorsilberniederschlag hinzugefügt und bis zum beginnenden Schmelzen erhitzt. Nach dem Erkalten über Schwefelsäure wurde der Porcellantiegel gewogen und aus dessen Gewichtszunahme nach Abzug der Filterasche das Chlor berechnet.

2) Durch Decantiren in einer Glasschale. - Eine genau gewogene Salzmenge wurde in oiner genau gewogenen Glasschale in heifsem, durch Salpetersãure angesäuertem Wasser gelöst und mit überschüssiger Silbernitratlösung versetzt. Sobald sich die über dem Chlorsilberniederschlag befindliche Flüssigkeit vollständig geklärt hatte, wurde sie vorsichtig mit einer Pipette abgezogen und der Niederschlag mit heifsem, durch Salpetersäure angesãuertem Wasser übergossen, die Flüssigkeit abermals. abgezogen und diefs so oft wiedurholt, bis die algezogene Flüssigkeit mit Salzsaure versetzt nicht mehr getrübt wurde. Letzteres war schon nach acht- bis neunmaligem Auswaschen erreicht. Der Niederschlag wurde 
nun in der Glasschale auf einem Wasserbade vollständig getrocknet und sodann auf einem Sandbade so lange vorsichtig erhitzt, bis das Chlorsilber zu schmelzen anfing. Nach dem Erkalten über Schwefelsäure wurde die Glasschale gewogen und aus der Gewichtszunahme das Chlor berechnet.

Ich habe mich von der genauen Uebereinstimmung beider Methoden durch vorherige wiederholte Versuche mit reinem Chlornatrium üherzeugt.

Das zur Fällung angewandte Silbernitrat wurde durch Auflōsen von reinem granulirtem Silber in reiner Salpetersüure' Eindampfen zur Krystallisation und Auswaschen der Krystalle mit Wasser erhalten.

Dit Salpetersäure wurde zuvor auf einen etwaigen Chlorgehalt geprüft, indern $50 \mathrm{CC}$. der Säure mit rinigen Tropfen Silbernitratlösung versetzt wurden. Die Flüssighteit blieb selbst nach 24 stündigen Sthen volikommen klar.

Die zur Wägung angewandten Gewichte wurden vorher genau unter einander verglichen.

Endlich wurde jedesmal die zur Auflosung bestimmte Menge Chlorcäsium so lange einer Temperatur von $150^{\circ} \mathrm{C}$. ausgesetzt, bis sich deren Gewicht vollständig constant zeigte.

I. 1,5825 Grm. des bei $150^{\circ} \mathrm{C}$. sich constant seigenden Chlorcalsiums gaben 1,351 (hlorsilber, demuach 0,3342195 Chlor oder 21,119 pC:,

daraus folgt : Atomgewicht des Cäsiums $=132,595$.

Die Bestimraung wurde in einer Glasschale susgeführt.

II. 1,3487 Grm. Chlorcäsium gaben 1,1501 Chlorsilber, demnach $0,28+52$ Chlor oder $21,096 \mathrm{pC}$,

daraus folgt : Atomgewicht des Cäsiums $=132,770$.

Die Bestimmung wurcie durch Filtration ausgeführt.

III. 1,188 Grm. Chlorcăsium gaben 1,0141 Chlorsilber, demnach 0,25087 Chlor oder 21,117 pC.,

daraus folgt : Atongewicht des Cäsiums $=132,611$.

Die Bestimmung wurdo in einer Glasschsle ausgeführt. 
IV. 1,2309 Grm. Chlorcksium gaben 1,051 Chlorsilber, demnach 0,260 Chlor oder 21,122 pC.,

daraus folgt : Atomgewicht des Cäsiuns $=132,585$.

Die Bestimmung wurdo in einer Glasschale ausgefürt.

Im Mittel dieser vier Bestimmangen, bei welchem Chlor $=35,5$ und silber $=108$ angenommen wurde, orhielt ich demnach 21,1135 pC. Chlor, daraus folgt :

Atomgewicht des Cäsiums $=132,627$ oder in runder Zahl 132,6.

Nimmt man Chlor $=35,46$ und Silher $=107,94$, so stellen sich folgende Zahlen heraus :

Bei Atomgewichtsbestimmung

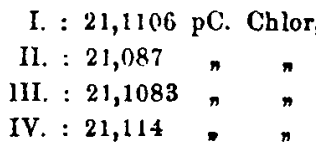

oder in Mittel dieser vier Versuche : Chlor $=21,104975$ pC., daraus berechuet sich dss

Atomgewicht des Cäsiums $=131,557$ oder in runder Zahl 132,6.

Bunsen*) fand im Mittel aus drei völlig übereinstimmenden Versuchen den Chlorgehalt des Chlorcäsiums $=21,0513$ pC., woraus das Atomgewicht des Cäsiums $=$ 132,99 oder in runder Zahl $=133$ folgt.

S. W. Johnson und O. D. Allen * fanden den Chlorgehalt des Chlorcäsiums im Mittel von vier fast völlig übereinstimmenden Versuchen $=21,045 \mathrm{pC}$, woraus das Atomgewicht des Cäsiums $=133,036$ oder in runder Zahl $=133$ folgt. Auch Mercer***) hat, wie Frankland ohne nähere Angabe mittheilt, für das Atomgewicht des Cäsiums die Zahl 133 gefunden.

\section{Atomgewichtsbestimmung des Rubidiums.}

Zur Darstellung von vollkommen reinem Chlorrubidium für die Atomgewichtsbestimmung des Rubidiums wurde nur

*) Pogg. Annalen 119, 1; Jahresber. f. Chemio u. 8. w. f. 1863, 187.

**) Journal fïr praktische Chemie 89, 154; Jahresbericht f. Chemio u. s. w. f. $1863,186$.

***) Chem. Nows 8, 18; Jabresber. f. Chemie o. B. w. f. $1863,189$. 
der vollkommen reine Rubidiumalaun genommen, d. h. jener, welcher im Spectralapparate nur die Rubidiumlinien zeigte.

Die Umwandlung dieses Alauns in Chlorrubidium geschah auf dieselbe Weise und mit Beobachtung aller jener Vorsichtsmafsregeln, welche ich für die Umwandlung des Cäsiumalauns in Chlorcäsium angegeben habe. Auch hier wurde jedesmal die zur Auflösung bestimmte Menge Chlorrubidium vor jeder Atomgewichtsbestimmung so lange einer Temperalur von $150^{\circ} \mathrm{C}$. ausgesetzt, bis sich das Gewicht des Salzes constant zeigte.

I. 1,4055 Grin. des bei $150^{\circ} \mathrm{C}$. sich constant zeigenden Chlorrulidiums gaben 1,6665 Chlorsilber, demnach 0,41227 Chlor oder 29,332 pC.,

daraus folgt : Atongewicht des Rubidiums $=85,5403$.

Die Bestimmung wurde in einer Glasschale susgeführt.

II. 1,8096 Grm. Chlorrubidiun gaben 2,1461 Chlorsilber, demnach 0,530916 Chlor oder 29,338 pC.,

daraus folgt : Atomgewicht des Rubidiums $=85,504$.

Die Bestimmung worde durch Filtration ausgefübrt.

III. 2,2473 Grna. Chlormbidiurs gaben 2,665 Chlorsilber, demnach 0,659285 Chlor oder 29,336 pC.,

daraus folgt : Atongewicht des Rubidiums $=85,511$.

Die Bestimmung wurde in einer Glasschale ausgeführt.

IV. $2,273 \mathrm{Grm}$. Chlorrubidium gaben 2,6946 Chlorsilber, demnach $0,66 € 608$ Chlor odex $29,328 \mathrm{pC}$,

daraus folgt : Atomgewicht des Rubidiums $=85,544$.

Die Bestimmnng wurde durch Filiration ausgeführt.

In Mittel dieser vier bestimmungen, bei welchem Chlor $=35,5$ und Silber $=108$ angenommen wurde, erbielt ich demnach 29,3335 pC. Chlor, daraus folgt :

Atomgewicht des Rubidiums $=85,52475$ oder in runder Zahl $=85,5$.

Nimmt man Chlor $=35,46$ und Silber $=107,94$, so stellen sich folgcnde Zahlen heraus : 
Bei Atomgewichtobestimmang I. : 29,3199 pC. Chlor,

$\begin{array}{lllll}n & n & \text { II. }: 29,3262 & n & n \\ n & * & \text { III. }: 29,3147 & n & n \\ n & n & \text { IV. }: 29,3241 & & n\end{array}$

oder im Mittel dieser vier Versuche : Chlor $=29,321225$ pC., darsus berechnet sich das

Atomgewicht des Rubidiums mit 85,476 oder in runder Zahl mit 85,5 .

Bunse I*) hat das Atomgewicht des Rubidiums abgeleitet aus dem Chlorgehalt des reinen Chlorrubidiums mit 85,36 und J. Piccard*\#) im Mittel aus vier Versuchen mit 85,41 gefunden.

Bei Gelegenheit der Aufsuchung geeigneter Reagentien zur Unterscheidung des Cäsiums, Rubidiums und Kaliums von rinander fand ich, dafs Pikrinsäure ebenso wie mit Kalium auch mit Rubidium und Cäsium geibe krystallinische Niederschläge erzeugt, welche namentlich in alkoholischen Flüssigkeiten schwer löslich sind. Aufserdem fand ich noch, dafs die Lösungen der Sulfate von schweren Metallen in verdünuter Schwefelsäure mit einer concentrirten Lösung von Cäsiumsulfat in mafsig verdünnter Schwefelsāure einen Niederschlag erzeugen, wie diefs auch bei den Lösungen der Chloride dieser Metalle in Salzsáure mit einer Lösung von Chlorcäsium in Salzsäure der Fall ist. Kalium- und Rubidiumsalze auf gleiche Weise behandelt zeigen diese Reaction nicht.

Giefsen, Laboratorium des Professors Will.

*) Jahresber. f. Chemie u. s. w. f. 1861, 175.

**) Daselbst, f. $1862,124$. 